\title{
Enhancing Efficiency and Robustness of a Photovoltaic Power System under Partial Shading
}

\author{
Yanzhi Wang ${ }^{1}$, Xue Lin ${ }^{1}$, Younghyun $\mathrm{Kim}^{2}$, Naehyuck Chang ${ }^{2}$, Massoud Pedram ${ }^{1}$ \\ ${ }^{1}$ University of Southern California, Los Angeles, CA USA \\ ${ }^{2}$ Seoul National University, Seoul, Korea \\ ${ }^{1}$ E-mail: \{yanzhiwa, xuelin, pedram $\} @$ usc.edu, ${ }^{2}$ E-mail: \{ yhkim, naehyuck $\} @$ elpl.snu.ac.kr
}

\begin{abstract}
Photovoltaic (PV) power systems have been widely applied in commercial and domestic facilities. Electrical energy storage (EES) systems are mandatory in standalone PV systems for continuous power supply. In this paper the efficiency and robustness enhancement methods for PV systems under partial shading have been investigated. Partial shading due to moving clouds and shadows of nearby obstacles on a PV module array causes significant efficiency degradation, since shaded and non-shaded PV modules have large discrepancy in their maximum power points (MPPs). Use of Individual charger for each PV module may mitigate the negative effect from partial shading. However, this method alone may still face severe energy efficiency degradation caused by i) the energy loss due to parasitic effects in the EES elements under variable incoming power from the PV modules and ii) the energy loss in each charger incurred by potentially high imbalance between its input and output voltages. This paper proposes three methods to enhance the PV system efficiency and robustness under partial shading: i) incorporation of a HEES (hybrid electrical energy storage) system into the PV system, ii) extension of the MPTT (maximum power transfer tracking) approach, and iii) dynamic PV module reconfiguration. The three proposed methods can be effectively combined together, yielding a significant efficiency gain ranging from $17.1 \%$ to $53.3 \%$ compared with the baseline systems.
\end{abstract}

\section{Keywords}

Photovoltaic system, partial shading, hybrid electrical energy storage system, dynamic reconfiguration

\section{Introduction}

Photovoltaic (PV) energy generation techniques have been investigated intensively in the past several decades, due to the continuously growing demand for renewable, environmental-friendly and cost-efficient energy sources. Thanks to the availability and abundance of the solar energy, various scales of PV power systems have been widely applied in commercial and domestic facilities. However, the solar energy is changing frequently according to the time of day and the surrounding weather/environment conditions, and therefore, standalone PV power systems typically require integrated electrical energy storage (EES) system. The primary goal of this paper is to investigate how to

*This research is sponsored in part by a grant from the National Science Foundation, and by the Brain Korea 21 Project, IC Design Education Center (IDEC), and Mid-career Researcher Program through NRF grant funded by the MEST (No. 2010-0017680). The ICT at Seoul National University provides research facilities for this study. improve the energy efficiency and robustness of a standalone PV power system, consisting of an array of PV modules, EES elements, and distributed chargers.

Conventional standalone PV systems generally have homogeneous EES systems consisting of a single type of batteries. On the other hand, recent work introduces hybrid EES (HEES) system [1][2][3] to leverage the efficiency. In this paper, we construct a HEES system with high-powercapacity EES arrays (supercapacitor arrays) together with the battery arrays and incorporate the HEES into the PV system. We apply a crossover filter to the irradiation and temperature profiles of the source PV modules, and thereby allow the battery arrays to steadily receive energy from the PV modules, while leave the spiky parts in the power supply to be dealt with by the supercapacitor arrays.

A PV module exhibits highly non-linear current-voltage (I-V) characteristics and mandates maximum power point tracking (MPPT) to pull out the maximum power from the module. Tracking the maximum power point (MPP) can be implemented in many ways such as the "perturb and observe" [4] and an incremental conductance approach [5]. Recently, it turns out that the traditional MPPT approach no longer guarantees maximum energy transferred into the EES elements due to significant power loss in the chargers. The maximum power transfer tracking (MPTT) method takes into account efficiency variation of the charger and achieves the optimal energy efficiency on the simple system of a single PV module charging a single supercapacitor [6]. However, researches are yet to be done for integrating the MPTT idea into more general PV systems consisting of multiple PV modules, multiple (perhaps hybrid) EES arrays, and a more complicated charge transfer interconnect (CTI).

Except for some desert areas, partial shading, which typically occurs due to moving clouds and shadows of nearby obstacles, may make each PV module in the PV module array have different solar irradiation strength. Partial shading not only reduces the maximum output power of the shaded PV module, but may also deviate other non-shaded PV modules (connected in series with the shaded one) from their MPPs, and thus the maximum output power of a partially shaded PV array may be much less than the sum of maximum output power values of all the PV modules in the array. In addition, partial shading may result in multiple power peaks in the power-voltage characteristics of an array of PV modules, and thus the MPPT (or MPTT) techniques must be improved to track a global optimal power point instead of a local optimal power point [7][8], which could increase the complexity of the PV system. The individual charger interface topology, i.e., each PV module connecting 
with an individual charger, has been proposed to prevent the shaded PV modules from severely degrading the performances of the non-shaded PV modules [9]. However, this individual charger interface method may still face severe efficiency degradation due to i) significant energy loss in the EES elements under variable incoming power from the PV modules and ii) potentially high energy loss in the chargers since partial shading may cause high imbalance between the input and output voltages of each charger.

In this paper, we call a PV system robust to partial shading if the energy efficiency is consistently high under various spatiotemporal-variant shading patterns among the PV modules. We consider two major energy conversion efficiency degradation factors: i) the energy loss due to parasitic effects in the EES elements and ii) the nonnegligible energy loss in the chargers, which is a function of their input and output voltages and currents. Energy loss due to i) is more significant when the incoming power from the PV modules is higher, while the EES elements have lower power capacity. Energy loss due to ii) becomes more distinctive if solar irradiance strength and state of charges (SoC's) of EES elements result in greater imbalance between the input and output voltages of each charger. Therefore, we propose the following three methods based on the individual charger interface topology for higher energy efficiency and robustness under partial shading: i) incorporation of HEES into a standalone PV system and developing a near-optimal HEES control algorithm, ii) extension of the MPTT approach, and iii) a novel dynamic balanced PV module reconfiguration method to enhance the charger efficiencies. The three methods can be effectively combined together, thereby yielding an energy efficiency improvement ranging from $17.1 \%$ to $53.3 \%$, compared with the baseline systems using traditional MPPT control, fixed PV module configurations, and homogeneous EES system.

\section{Component Models}

\subsection{PV Model and Characterization}

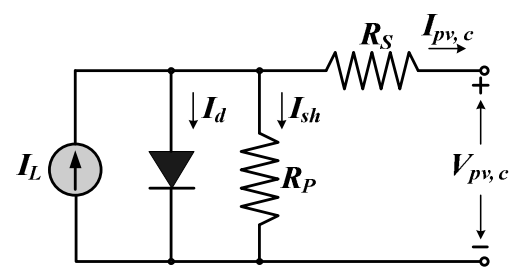

Figure 1: Equivalent circuit model of a PV cell

As mentioned before, the energy source of the proposed system is a PV array, consisting of multiple PV modules. Each PV module again consists of a number of PV cells, connected with each other in a balanced series and parallel configuration. Consider a PV module consisting of $m \times n$ PV cells, where $m$ is the number of PV cells connected in series and $n$ is the number of PV cells connected in parallel. If we use $V_{p v}$ and $I_{p v}$ to denote the output voltage and current of the PV module, respectively, and use $V_{p v, c}$ and $I_{p v, c}$ to denote the output voltage and current of a single PV cell inside that module, respectively, we shall have:

$$
V_{p v}=m \cdot V_{p v, c}, \quad I_{p v}=n \cdot I_{p v, c} .
$$

A typical equivalent circuit model of a PV cell is shown in Figure 1, with I-V characteristics given by:

$$
\begin{aligned}
& I_{p v, c}=I_{L}-I_{d}-I_{s h}= \\
& \left.I_{L}(G)-I_{0}(T)\left(e^{\left(V_{p v, c}+I_{p v, c} \cdot R_{S}\right.}\right) \cdot \frac{q}{A k T}-1\right)-\frac{V_{p v, c}+I_{p v, c} \cdot R_{S}}{R_{p}},
\end{aligned}
$$

where $I_{L}(G)$ and $I_{0}(T)$ are given by:

$$
\begin{gathered}
I_{L}(G)=\frac{G}{G_{S T C}} \cdot I_{L}\left(G_{S T C}\right) \\
I_{0}(T)=I_{0}\left(T_{S T C}\right) \cdot\left(\frac{T}{T_{S T C}}\right)^{3} \cdot e^{\frac{q E_{g}}{A k} \cdot\left(\frac{1}{T_{S T C}}-\frac{1}{T}\right)} .
\end{gathered}
$$

We denote the parameters in (2) (4) such that $G$ is the irradiance level; $T$ is the cell temperature; $q$ is the charge of the electron; $E_{g}$ is the energy bandgap and $k$ is the Boltzmann's constant. STC stands for standard test condition in which irradiance level is $1000 \mathrm{~W} / \mathrm{m}^{2}$ and the cell temperature is $25{ }^{\circ} \mathrm{C}$. Besides, there are still five unknown parameters, commonly not provided by manufacturers, yet to be determined, as listed below.

- $I_{L}\left(G_{S T C}\right)$ : photo-generated current at STC.

- $I_{0}\left(T_{S T C}\right)$ : dark saturation current at STC.

- $R_{s}$ : PV cell series resistance.

- $R_{p}$ : PV cell parallel (shunt) resistance.

- $A$ : diode ideality factor.

We adopt the method proposed in [10] which could effectively extract the above-mentioned five unknown parameters from the measured PV cell I-V curve.

\subsection{Charger}

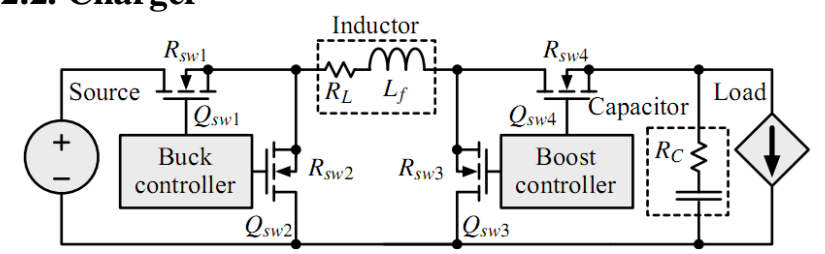

Figure 2: Buck-boost converter architecture

A charger is a PWM (pulse width modulation) buckboost switching converter which regulates its output current into a desired value, with model shown in Figure 2. The input voltage, input current, output voltage and output current of the charger are denoted by $V_{\text {in }}, I_{\text {in }}, V_{\text {out }}$, and $I_{\text {out }}$, respectively. We use $P_{\text {conv }}$ to denote the power loss of the charger, which consists of the conduction loss, the switching loss and the controller loss [3], and we have:

$$
V_{\text {in }} \cdot I_{\text {in }}=P_{\text {conv }}+V_{\text {out }} \cdot I_{\text {out }} \text {. }
$$

Based on the relationship between $V_{\text {in }}$ and $V_{\text {out }}$, the charger has two operating modes: the buck mode if $V_{\text {in }}>$ $V_{\text {out }}$ and the boost mode otherwise. When the charger is in the buck mode, its power loss $P_{\text {conv }}$ is given by

$$
\begin{aligned}
P_{\text {conv }} & =I_{\text {out }}^{2} \cdot\left(R_{L}+D \cdot R_{s w 1}+(1-D) \cdot R_{s w 2}+R_{s w 4}\right) \\
& +\frac{(\Delta I)^{2}}{12}\left(R_{L}+D \cdot R_{s w 1}+(1-D) \cdot R_{s w 2}+R_{s w 4}+R_{C}\right) \\
& +V_{\text {in }} \cdot f_{s} \cdot\left(Q_{s w 1}+Q_{s w 2}\right)+V_{\text {in }} \cdot I_{\text {controller }},
\end{aligned}
$$
where $D=V_{\text {out }} / V_{\text {in }}$ is the PWM duty ratio and $\Delta I=V_{\text {out }}$. $(1-D) /\left(L_{f} \cdot f_{s}\right)$ is the maximum current ripple; $f_{s}$ is the switching frequency; $I_{\text {controller }}$ is the current flowing into micro-controller; $R_{L}$ and $R_{C}$ are internal series resistances of the inductor $L$ and the capacitor $C$, respectively; $R_{S w i}$ and $Q_{\text {swi }}$ are the turn-on resistance and gate charge of the $i$-th MOSFET switch shown in Figure 2, respectively. 
The charger power loss $P_{\text {conv }}$ in the boost mode is:

$P_{\text {conv }}=\left(\frac{I_{\text {out }}}{1-D}\right)^{2}$.

$\left(R_{L}+D \cdot R_{s w 3}+(1-D) \cdot R_{s w 4}+R_{s w 1}+D(1-D) \cdot R_{C}\right)$

$+\frac{(\Delta I)^{2}}{12}\left(R_{L}+D \cdot R_{s w 3}+(1-D) R_{s w 4}+R_{s w 1}+(1-D) R_{C}\right)$

$+V_{\text {out }} \cdot f_{s} \cdot\left(Q_{s w 3}+Q_{s w 4}\right)+V_{\text {in }} \cdot I_{\text {controller }}$,

where $D=1-V_{\text {in }} / V_{\text {out }}$ and $\Delta I=V_{\text {in }} \cdot D /\left(L_{f} \cdot f_{s}\right)$.

\subsection{EES Element Array}

Without loss of generality, this paper addresses the PV system optimization with two kinds of representative EES elements, Li-ion battery and supercapacitor, to deliver the main concepts. We use $V_{\text {array }}^{O C}(t)$ and $V_{\text {array }}^{C C}(t)$ to denote the open circuit terminal voltage (OCV) and closed circuit terminal voltage $(\mathrm{CCV})$ of an EES array at time $t$, respectively, and use $I_{\text {array }}(t)$ to denote the array charging/discharging current. The $V_{\text {array }}^{O C}(t)$ and $V_{\text {array }}^{C C}(t)$ values are typically not equal to each other due to the effect of internal resistances and capacitances in an EES array. Moreover, we use $V_{S O C}(t)$ as the voltaic representation of the EES array SoC at time $t$, defined as the ratio of the charge stored in an EES array to the total charge when the array is fully charged. In general, the OCV value $V_{\text {array }}^{O C}(t)$ is a monotonically increasing function of the SoC $V_{S O C}(t)$.

We use an electronic circuit model in [11] for the Li-ion battery model. In this model, the relation between the battery array $\mathrm{OCV} V_{\text {array }}^{O C}(t)$ and $\mathrm{CCV} V_{\text {array }}^{C C}(t)$ is given by:

$$
V_{\text {array }}^{C C}(t)=V_{\text {array }}^{O C}(t)+V_{t l}(t)+V_{t s}(t)+I_{\text {array }}(t) \cdot R_{s},
$$

where $V_{t l}(t)$ and $V_{t s}(t)$ are the voltage drops across the array internal capacitances, and $R_{S}$ is the array internal series resistance. Moreover, the OCV-SoC relationship for a battery array is given by the following non-linear function:

$$
\begin{gathered}
V_{\text {array }}^{O C}(t)=b_{1} \cdot e^{b_{2} \cdot V_{S O C}(t)}+b_{3} \cdot V_{S O C}^{3}(t)+b_{4} \cdot V_{S O C}^{2}(t)+ \\
b_{5} \cdot V_{S O C}(t)+b_{6},
\end{gathered}
$$

where those $b_{i}$ are empirically determined parameters [12].

On the other hand, the internal series resistance of supercapacitor is negligible, i.e., $V_{\text {array }}^{C C}(t) \approx V_{\text {array }}^{O C}(t)$, and the OCV $V_{\text {array }}^{O C}(t)$ is a linear function of the SoC $V_{S O C}(t)$.

The rate capacity effect of Li-ion batteries specifies that the charging and discharging efficiencies of a battery array decrease with the increasing of charging and discharging currents, respectively. We focus on the charging efficiency degradation scheme of battery arrays, since the destination EES arrays are being charged by multiple PV modules in the target system. More precisely, the Peukert's Law [13] describes that the charging efficiency of a battery array, as a function of the charging current $I_{\text {array }}(t)$, is given by

$$
\eta_{\text {rate }}\left(I_{\text {array }}(t)\right)=k_{c} /\left(I_{\text {array }}(t)\right)^{\alpha_{c}},
$$

where $k_{c}$ and $\alpha_{c}$ are constants known a priori. The rate capacity effect of a supercapacitor array is negligible, i.e., both charging and discharging efficiencies are equal to one.

\section{PV Power System}

\subsection{PV Power System Architecture}

Figure 3 shows the architecture of the PV system with homogeneous EES arrays (battery arrays) for energy storage, named Homogeneous EES-Based System; while
Figure 4 shows the architecture of the proposed PV system with HEES element arrays for energy storage, named HEESBased System. Both systems consist of a power source, an energy storage (sub-) system, a single-wire CTI, and chargers. The power source of both Homogeneous EESBased System and the proposed HEES-Based System consists of a set of $M \mathrm{PV}$ modules from a single large PV array. Certain control mechanisms, e.g., MPPT, MPTT, and/or module reconfiguration, can be integrated into each PV module for efficiency enhancement. The PV modules are connected to the CTI via distributed (individual) chargers. This "individual charger interface" structure can mitigate the negative effect on the output power of the whole PV array due to partial shading. On the right-hand side of the CTI, the energy storage system in Homogeneous EES-Based System is comprised of (multiple) homogeneous EES arrays connected via charging control chargers and discharging control chargers to the CTI. In contrast, the energy storage system in the HEES-Based System consists of multiple heterogeneous EES arrays with complementary characteristics connected via charging control chargers and discharging control chargers to the CTI. Each EES array is composed of multiple homogeneous EES elements as a typical single EES element has a small energy capacity. We aim to convey the idea of introducing a HEES system for efficiency enhancement, by using a two-array architecture, i.e., one battery array and one supercapacitor array. Thus the Homogeneous EES-Based System only contains a single battery array (which is the same as the battery array in the HEES-Based System) for fair comparison.

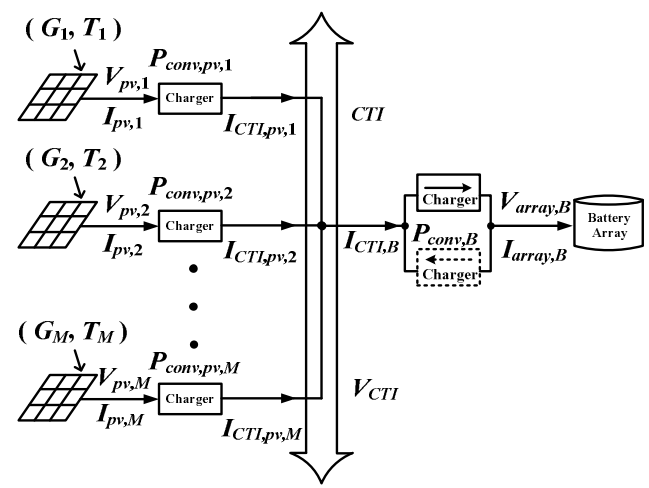

Figure 3: Architecture of the homogeneous EES-based PV system (Homogeneous EES-Based System)

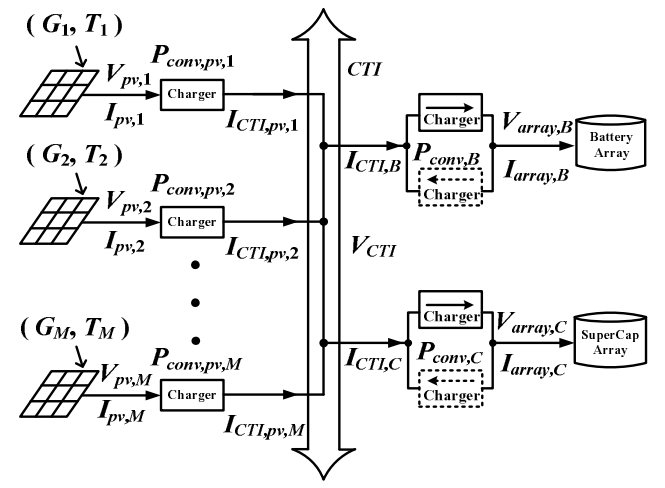

Figure 4: Architecture of the proposed HEES-based PV system (HEES-Based System) 
At time instance $t$, we use $V_{p v, i}(t)$ and $I_{p v, i}(t)$ to denote the output voltage and current of the $i$-th $(1 \leq i \leq M)$ source PV module, respectively. Obviously, the relationship between $V_{p v, i}(t)$ and $I_{p v, i}(t)$ values, as given in (1) (4), depends on the irradiance level $G_{i}(t)$ and the module temperature $T_{i}(t)$ on the $i$-th PV module at time $t$, as well as the configuration (the number of series and parallel connections of PV cells) of the $i$-th PV module. Note that $V_{p v, i}(t)$ and $I_{p v, i}(t)$ also serve as the input voltage and current of the charger connecting the $i$-th PV module to the CTI, respectively. On the other hand, the output voltage and current of that charger are denoted by $V_{C T I}(t)$ (the CTI voltage) and $I_{C T I, p v, i}(t)$, respectively, and its power loss is denoted by $P_{c o n v, p v, i}(t)$. The $P_{\text {conv }, p v, i}(t)$ value is a function of the $V_{p v, i}(t), I_{p v, i}(t), V_{C T I}(t)$ and $I_{C T I, p v, i}(t)$ values, as shown in Section 2.2, and we have:

$$
V_{p v, i}(t) \cdot I_{p v, i}(t)=P_{c o n v, p v, i}(t)+V_{C T I}(t) \cdot I_{C T I, p v, i}(t),
$$

by the energy conservation law.

For the battery array in the Homogeneous EES-Based System or the HEES-Based System, we use $V_{\text {array,B }}^{O C}(t)$ and $V_{\text {array, } B}^{C C}(t)$ to denote the OCV and CCV of the battery array at time $t$, respectively, and their relationship is given in Section 2.3. The current between the battery array and its corresponding chargers is denoted by $I_{\text {array,B }}(t)$, while the current between CTI and the corresponding chargers for the battery array is denoted by $I_{C T I, B}(t)$. In the HEES-Based System, we make the battery array steadily and continuously receive energy from the PV modules. Therefore the discharging control charger of the battery array is always turned off, and we have $I_{\text {array,B }}(t) \geq 0$ and $I_{C T I, B}(t) \geq 0$. Obviously these two inequalities also hold for the Homogeneous EES-Based System where there is only one battery array for energy storage. Moreover, the power loss value of the corresponding charging control charger for the battery array is denoted by $P_{c o n v, B}(t)$, which is a function of the $V_{C T I}(t), I_{C T I, B}(t), V_{\text {array, } B}^{C C}(t)$, and $I_{\text {array }, B}(t)$ values, as shown in Section 2.2. We have:

$$
V_{C T I}(t) \cdot I_{C T I, B}(t)=P_{\text {conv }, B}(t)+V_{\text {array }, B}^{C C}(t) \cdot I_{\text {array }, B}(t),
$$

by the energy conservation law. Similar notations can also be applied to the supercapacitor array in the HEES-Based System, as illustrated in Figure 4. However, for the supercapacitor array, the currents $I_{\text {array, } C}(t)$ and $I_{C T I, C}(t)$ can be positive (if power flows from CTI into the supercapacitor array), negative (if power flows from the supercapacitor array into the CTI), or zero. The variable $P_{\text {conv }, C}(t)$ actually denotes the power loss of either the charging control charger or the discharging control charger of the supercapacitor array. Those two chargers cannot be turned on at the same time since an EES array cannot be simultaneously charged and discharged.

Finally, the current values flowing into and out of the CTI satisfy the Kirchhoff's law, i.e.,

$$
\sum_{i=1}^{M} I_{C T I, p v, i}(t)=I_{C T I, B}(t)+I_{C T I, C}(t),
$$

where in Homogeneous EES-Based System $I_{C T I, C}(t)=0$.

\subsection{Problem Statement}

Let the system (Homogeneous EES-Based System or HEES-Based System) operation start at time $T_{0}$. The initial SoC of the battery array, denoted by $\left.V_{S O C, B}(t)\right|_{t=T_{0}}$, is given, and thus its initial OCV, $\left.V_{\text {array, } B}^{O C}(t)\right|_{t=T_{0}}$, can be derived using (9). On the other hand, the initial SoC (and also OCV) of the supercapacitor array in the HEES-Based System is zero (fully discharged), i.e., $\left.V_{S O C, C}(t)\right|_{t=T_{0}}=0$ and $\left.V_{\text {array, } C}^{O C}(t)\right|_{t=T_{0}}=0$, for more realistic operating scenarios. In fact, supercapacitors are not suitable for long-term energy storage due to its high self-discharge rate. The solar irradiation is available during the time period $\left[T_{0}, T_{a}\right]$. Moreover, there exists a system operation deadline time $T_{d}$ $\left(T_{d} \geq T_{a}\right)$, by which time system operations must finish.

The PV systems in this paper run in an online manner, i.e., the system controller is not aware of the irradiation levels and temperatures among PV modules in the future. More specifically, at any time instance $t \in\left[T_{0}, T_{d}\right]$ during system operation, the irradiance level $G_{i}(t)$ and the module temperature $T_{i}(t)$ of the $i$-th $(1 \leq i \leq M)$ PV module are available (note that $G_{i}(t)=0$ when $t \in\left[T_{a}, T_{d}\right]$ ), and therefore the PV module output voltage $V_{p v, i}(t)$ and output current $I_{p v, i}(t)$ satisfy (1) (4) (in which $G \leftarrow G_{i}(t)$ and $\left.T \leftarrow T_{i}(t)\right)$. They also depend on the configuration, i.e., numbers of series and parallel connections of PV cells, of the $i$-th PV module. Moreover, at that time $t$, the battery array and supercapacitor array (in the HEES-Based System) SoC values can be calculated via:

$$
\begin{aligned}
V_{S O C, B}(t) & =V_{S O C, B}\left(T_{0}\right) \\
& +\frac{1}{Q_{B, \text { full }}} \int_{T_{0}}^{t} I_{\text {array }, B}(\tau) \cdot \eta_{\text {rate }, B}\left(I_{\text {array }, B}(\tau)\right) \mathrm{d} \tau, \\
V_{S O C, C}(t) & =V_{S O C, C}\left(T_{0}\right)+\frac{1}{Q_{C, \text { full }}} \int_{T_{0}}^{t} I_{\text {array }, C}(\tau) \mathrm{d} \tau,
\end{aligned}
$$

where $Q_{B, f u l l}$ and $Q_{C, \text { full }}$ denote the full charge of the battery array and the supercapacitor array, respectively. The OCV values of the battery array and the supercapacitor array can be calculated based on the SoC values via the OCV-SoC relation for battery (9) and for supercapacitor (linear function.) In order for efficiency optimization, the system controller controls the PV module operation points $\left(V_{p v, i}(t)\right.$, $\left.I_{p v, i}(t)\right)$ for $1 \leq i \leq M$ (through controlling the currents $I_{C T I, p v, i}(t)$ for $\left.1 \leq i \leq M\right)$, the CTI voltage $V_{C T I}(t)$, as well as the battery and supercapacitor array currents, $I_{\text {array,B }}(t)$ and $I_{\text {array, }}(t)$, respectively, at time $t$, based on the abovementioned environmental parameters and EES elements state parameters (SoC, OCV, etc.).

The objective of both the Homogeneous EES-Based System and the HEES-Based System is to store the maximum amount of energy inside the battery array within deadline time $T_{d}$, i.e., we maximize

$$
\int_{T_{0}}^{T_{d}} V_{\text {array }, B}^{O C}(t) \cdot I_{\text {array }, B}(t) \cdot \eta_{\text {rate }, B}\left(I_{\text {array }, B}(t)\right) \mathrm{d} t .
$$

This objective is equivalent to the maximization of the energy conversion efficiency within the deadline time $T_{d}$. The energy conversion efficiency is defined as the ratio of the total energy stored into the battery array during time period $\left[T_{0}, T_{d}\right]$ to the maximum available energy generated 
by all the source PV modules during time period $\left[T_{0}, T_{a}\right]$, when each PV module works at its own MPP at any time $t \in\left[T_{0}, T_{a}\right]$. The battery array has relatively large energy capacity and low self-discharge rate, and is therefore more suitable for long-term energy storage.

\section{Proposed Efficiency Enhancing Methods}

We first specify the baseline system, as all the proposed efficiency enhancement methods can be viewed as improvements on the baseline system. The baseline system uses the Homogeneous EES-Based System architecture, with a fixed (and predefined) CTI voltage, MPPT control of each PV module, and no PV module reconfiguration. Obviously, the baseline system should have the lowest energy conversion efficiency and the worst robustness under various input irradiance and temperature profiles, as revealed in experimental results.

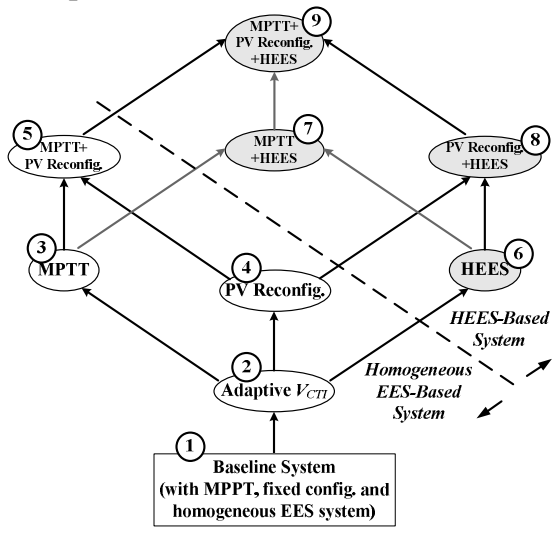

Figure 5: The proposed enhancement methods

Figure 5 shows the evolution of the baseline PV system equipped with different (combination of) efficiency enhancement methods, in which arrows denote improvements and nodes denote corresponding systems. Although not discussed before, the most basic efficiency enhancement method is named adaptive CTI voltage. We discuss the adaptive CTI voltage enhancement on the baseline system in the following. The PV operation points $\left(V_{p v, i}(t), I_{p v, i}(t)\right)$ for $1 \leq i \leq M$ at each time $t$ are known since the PV modules with fixed configurations work on their MPPs. Therefore for each CTI voltage value $V_{C T I}(t)$, we calculate the CTI input currents $I_{C T I, p v, i}(t)$ for $1 \leq i \leq$ $M$ from (11). Furthermore, since the target system (baseline system with adaptive CTI voltage) uses the Homogeneous EES-Based System architecture which only contains battery array for energy storage, the battery array charging current $I_{\text {array }, B}(t)$ can be calculated using (12) and (13) in which $I_{C T I, C}(t)=0$. Therefore, the received power by the battery array, defined as $P_{\text {array }, B}^{\text {recv }}(t)=V_{\text {array }, B}^{O C}(t) \cdot I_{\text {array }, B}(t)$. $\eta_{\text {rate }, B}\left(I_{\text {array }, B}(t)\right)$, is a function of the $V_{C T I}(t)$ value, denoted by $P_{\text {array, } B}^{\text {recr }}\left(V_{C T I}(t)\right)$. Based on the above calculation, the adaptive CTI voltage method finds the most suitable CTI voltage $V_{C T I}(t)$ value at any time instance $t \in\left[T_{0}, T_{a}\right]$ during system operation, such that the received power by the battery at that time $t, P_{\text {array, } B}^{\text {recv }}\left(V_{C T I}(t)\right)$, can be maximized. The system stops operation when $t \in\left[T_{a}, T_{d}\right]$, because there is no energy input from PV modules (this claim only holds for the Homogeneous EES-Based System architecture.) Besides, maximizing the received power $P_{\text {array, } B}^{\text {recv }}\left(V_{C T I}(t)\right)$ is equivalent to maximizing the battery array charging current $I_{\text {array, } B}(t)$, and we shall use the two objectives interchangeably. Finding the optimal $V_{C T I}(t)$ value can be accelerated by the ternary search algorithm, using the quasi-concavity of the function $P_{\text {array, } B}^{r e c v}\left(V_{C T I}(t)\right)$. The ternary search converges in logarithmic time with respect to the $V_{C T I}(t)$ precision. The detailed algorithm, named basic CTI voltage selection (B-VCTI) algorithm, for outputting the set of system control variables $\mathbf{C V}(t)=$ $\left\{\left(V_{p v, i}(t), I_{p v, i}(t)\right)\right.$ for $\left.1 \leq i \leq M, V_{C T I}(t), I_{\text {array }, B}(t)\right\}$ at time $t \in\left[T_{0}, T_{a}\right]$, is given in Algorithm 1 .

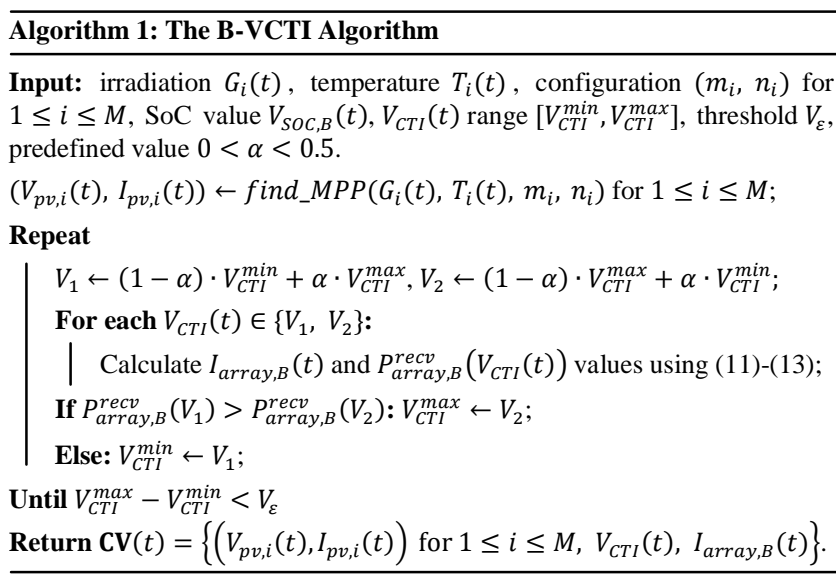

The $m_{i}$ and $n_{i}$ values used in Algorithm 1 denote the numbers of PV cells connected in series and in parallel in the $i$-th PV module, respectively. These values are fixed since we do not allow dynamic PV module reconfiguration here. The function find_MPP $\left(G_{i}(t), T_{i}(t), m_{i}, n_{i}\right)$ used in Algorithm 1 finds the MPP operation point of the $i$-th PV module given the solar irradiance $G_{i}(t)$, the temperature $T_{i}(t)$, and the PV module configuration $\left(m_{i}, n_{i}\right)$.

As illustrated in Figure 5, we propose further energy efficiency enhancement methods, including MPTT, dynamic PV module reconfiguration, and HEES, all based on the above-described adaptive CTI voltage enhancement method. These three enhancement methods can be effectively combined with each other, thereby yielding a total of seven types of enhanced PV systems over the baseline system with adaptive CTI voltage method. The HEES-Based System architecture shown in Figure 4 is the architecture support of the "HEES" efficiency enhancement method.

\subsection{MPTT Extension}

We extend the MPTT method, originally proposed in [6], in the multiple-PV-module system setting. The motivation of MPTT is that for each PV module, the traditional MPPT approach, i.e., the PV module works at its MPP, will not guarantee maximum energy transferred into the EES elements. The MPTT idea can be effectively integrated with the above-mentioned adaptive CTI voltage method. For any given CTI voltage $V_{C T I}(t)$ at time $t$, we can apply the MPTT idea to find the optimal operation point $\left(V_{p v, i}(t), I_{p v, i}(t)\right)$ (which may not be the MPP) of each $i$-th $(1 \leq i \leq M) \mathrm{PV}$ module so that its corresponding CTI input current 
$I_{C T I, p v, i}(t)$ can be maximized. The optimal $V_{C T I}(t)$ value can be obtained using the ternary search method. Finding the optimal PV operation point $\left(V_{p v, i}(t), I_{p v, i}(t)\right)$ of each $i$-th $(1 \leq i \leq M) \mathrm{PV}$ module to optimize the $I_{C T I, p v, i}(t)$ value for given $V_{C T I}(t)$ becomes a necessary condition of the overall efficiency maximization problem, i.e., maximizing the $P_{\text {array,B }}^{r e c v}\left(V_{C T I}(t)\right)$ value at time $t$. This is similar to the subproblem structure in a dynamic programming problem. The $M$ MPTT subproblems, one for each PV module, are independent with each other, and thus can be implemented via distributed PV module controllers. The detailed algorithm combining the adaptive $V_{C T I}(t)$ method with the MPTT idea for overall efficiency maximization at each time $t$, named the MPTT-based CTI voltage selection (M-VCTI) algorithm, is given in Algorithm 2.

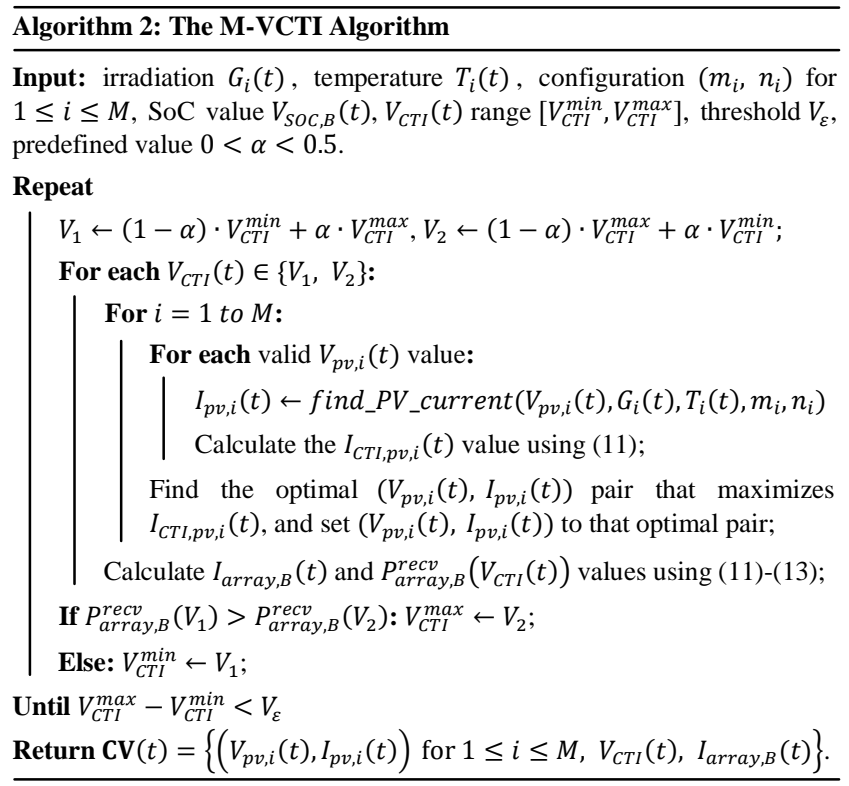

\subsection{Dynamic PV Module Reconfiguration}

We propose a novel dynamic balanced PV module reconfiguration method to find the "best match" between the input and output voltages of each charger in the PV system. This further mitigates the negative effect of the charger input and output voltage level imbalance. We first define a balanced configuration of a PV module consisting of $N$ identical PV cells to be the arrangement of cells in which there are $m$ sub-modules connecting in series. Each submodule consists of a set of $n$ cells connected in parallel. Obviously we have $N=m \times n$. The $N$-cell PV module can be organized into various balanced configurations and the number of possible configurations is equal to the number of bi-factor decompositions of $N$. We use $(m, n)$ to denote a configuration with $m$ cells in series and $n$ cells in parallel.

The proposed balanced PV module reconfiguration method requires a reconfigurable PV module structure as shown in Figure 6. The proposed reconfigurable PV module is composed of $N$ PV cells, and each cell has three switches: one series switch (S-switch) and two parallel switches (Pswitches) except for the last cell. The P-switches connect cells in parallel into sub-modules, whereas the S-switches connect the sub-modules in series. The $i$-th cell is associated with its S-switch, denoted by $S_{S, i}$. The two P-switches are denoted by $S_{P T, i}$ and $S_{P B, i}$. If $S_{S, i}$ is open, $S_{P T, i}$ and $S_{P B, i}$ are closed. Figure 7 shows the balanced reconfiguration of a four-cell reconfigurable PV module $(N=4)$.

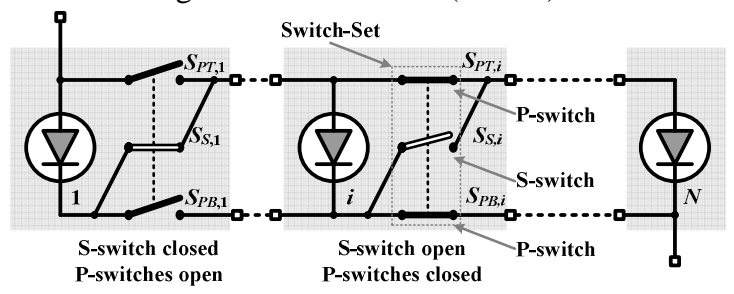

Figure 6: Reconfigurable architecture of $N$-cell PV module

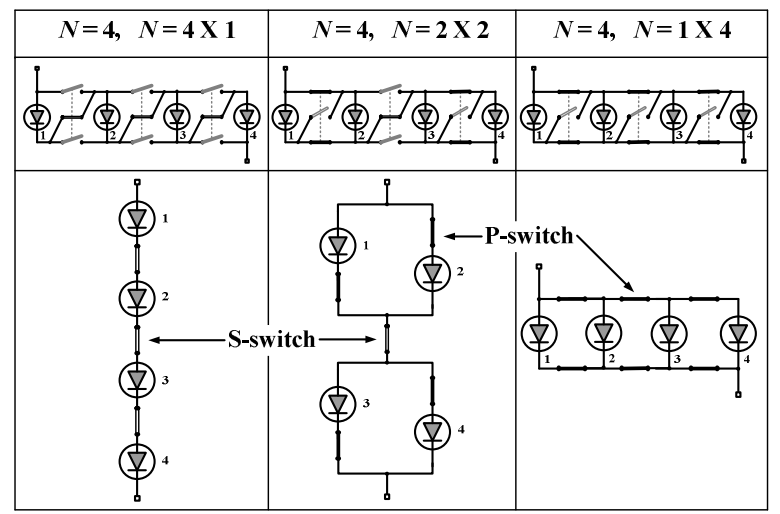

Figure 7: Reconfiguration of a four-cell PV module

The way that dynamic PV module reconfiguration can be integrated with adaptive $V_{C T I}(t)$ method is similar as the MVCTI algorithm shown in Algorithm 2, that is, for any given $V_{C T I}(t)$ value at time $t$, we search all possible balanced configurations of each $i$-th $(1 \leq i \leq M)$ PV module (each PV module works at its MPP) and find its best-suited configuration such that the corresponding CTI input current $I_{C T I, p v, i}(t)$ can be maximized. Then the optimal $V_{C T I}(t)$ value can be obtained using the ternary search method. The detailed algorithm for maximizing overall energy efficiency at time $t$, named the reconfiguration-based CTI voltage selection (R-VCTI) algorithm, is similar to Algorithm 2 and therefore not presented in this paper due to space limitation.

Integrating both the dynamic PV module reconfiguration and MPTT methods with the adaptive $V_{C T I}(t)$ method will yield further enhancement. At each time $t$, the system controller will find both the best-suited balanced configuration $\left(m_{i}(t), n_{i}(t)\right)$ and the optimal operating point $\left(V_{p v, i}(t), I_{p v, i}(t)\right)$ (based on the best-suited configuration, may not be MPP) of each $i$-th source PV module, as well as the optimal CTI voltage value $V_{C T I}(t)$. To avoid the time complexity arisen from determining the best-suited configuration and the optimal operation point at the same time for each PV module, we provide a nearoptimal solution of first finding the near-optimal balanced configuration of each PV module by assuming that it works at the MPP, and then find the optimal MPTT operating point of that PV module with the above-determined near-optimal configuration, for each given $V_{C T I}(t)$ value. Obviously, the optimal $V_{C T I}(t)$ value can be determined using ternary search, similar as before. The detailed algorithm for overall efficiency maximization at time $t$, named the MPTT and 
reconfiguration-based CTI voltage selection (MR-VCTI) algorithm, is given in Algorithm 3.

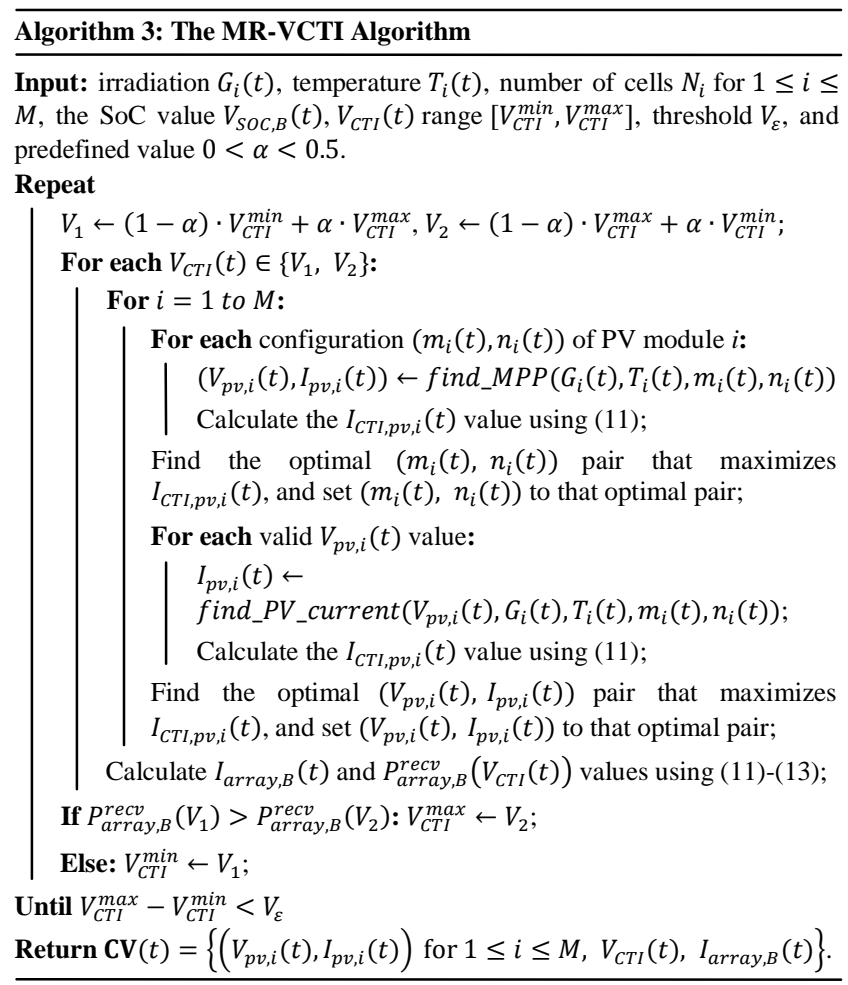

\subsection{HEES System Control Algorithm}

We focus on the near-optimal HEES control algorithm development in this section. The algorithm determines how to control the battery and supercapacitor array currents, $I_{\text {array }, B}(t)$ and $I_{\text {array }, C}(t)$, respectively, at each time $t \in\left[T_{0}, T_{d}\right]$, so that (16) can be maximized. We introduce a HEES enhancement method from the baseline system with an adaptive CTI voltage (shown in Figure 5). We illustrate the HEES method based on MPPT control and fixed PV module configurations. The MPTT and dynamic PV module reconfiguration methods can also be effectively integrated with the "HEES" method and yield a higher efficiency gain.

The underlying idea of the proposed HEES control algorithm is to apply a crossover filter to the solar irradiation and temperature profiles on the PV modules. This allows the battery array to receive the PV modules energy steadily and continuously, and make the high-frequency components in the power source profile handled by the supercapacitor array. The proposed HEES control algorithm consists of three steps: 1) initial charging of the supercapacitor array, 2) charging the battery array using the supercapacitor array as buffer, and 3) migrating charge from the supercapacitor array to the battery array.

1) Step One:

Since the initial SoC of the supercapacitor array is zero, we should charge the supercapacitor array from the beginning of system operation until its SoC reaches a predefined value. This enables us to use the supercapacitor array as a charge buffer during the battery array charging process. The initial charging phase finishes at time $T_{e}<T_{a}$, which is not known to the system controller in advance. We shall maximize the energy receiving rate of the supercapacitor array, defined as $P_{\text {array, } C}^{r e c v}(t)=V_{\text {array }, C}^{O C}(t)$. $I_{\text {array, } C}(t)$, at any time $t \in\left[T_{0}, T_{e}\right]$ to achieve the optimal initial charging. We can prove that maximizing the energy receiving rate $P_{\text {array, }}^{r e c v}(t)$ is equivalent to maximizing the supercapacitor array current $I_{\text {array, } C}(t)$. We add a new control variable $I_{\text {array,C }}(t)$ to Algorithm 1 (the B-VCTI algorithm) for the supercapacitor array power optimization, and set $I_{\text {array }, B}(t)=0$. We set $I_{\text {array }, C}(t)$ also to be the optimization objective of the modified B-VCTI algorithm. The system controller executes the modified B-VCTI algorithm at each time $t \in\left[T_{0}, T_{e}\right]$. Details of the algorithm modification are omitted due to space limitation. The MPTT and dynamic PV module reconfiguration methods can be incorporated into the modified B-VCTI algorithm for charging supercapacitor arrays, similar to the M-VCTI, RVCTI, and MR-VCTI algorithms.

\section{2) Step Two:}

The second and most important step of the proposed HEES control algorithm takes place during the time period $\left[T_{e}, T_{a}\right]$. In this step the supercapacitor array acts as a buffer that filters out the high-frequency components of the PV input power and provides steady battery charging operation. The motivation of this step is that the power loss in lowpower-capacity EES elements (e.g., batteries) due to the parasitic effects is a superlinear function of the energy incoming rate, and therefore it is desirable to let such EES elements with low power capacity receive the steady part of the incoming power. Based on this motivation, we apply a causal crossover filter on the solar irradiation profile $G_{i}(t)$ and temperature profile $T_{i}(t)$ of each $i$-th $(1 \leq i \leq M)$ PV module to separate the high frequency components and the low frequency components in such profiles. We use $\hat{G}_{i}(t)$ and $\widehat{T}_{i}(t)$ to denote the low frequency component outputs of the crossover filter on the irradiation and temperature profiles, respectively. The $\hat{G}_{i}(t)$ and $\hat{T}_{i}(t)$ values for $1 \leq i \leq M$ at each time $t \in\left[T_{e}, T_{a}\right]$ can be calculated as the moving averages of the past solar irradiation and temperature profiles, respectively.

Next we find the near-optimal target charging current for the battery array, denoted by $I_{\text {array }, B}^{\operatorname{tar}}(t)$, at each time $t \in\left[T_{e}, T_{a}\right]$, based on the derived $\widehat{G}_{i}(t)$ and $\widehat{T}_{i}(t)(1 \leq i \leq$ $M)$ values. We first maximize the battery array charging current $I_{\text {array,B }}(t)$ value through effectively finding the optimal CTI voltage $V_{C T I}(t)$, assuming that the solar irradiation and temperature on the each $i$-th $(1 \leq i \leq M) \mathrm{PV}$ module are given by $\widehat{G}_{i}(t)$ and $\widehat{T}_{i}(t)$, respectively. We do not consider the supercapacitor array here. We carry out this step through performing Algorithm 1 by setting $G_{i}(t) \leftarrow$ $\hat{G}_{i}(t)$ and $T_{i}(t) \leftarrow \widehat{T}_{i}(t)$ in the algorithm. Then we set $I_{\text {array }, B}^{\text {tar }}(t)$ to be the obtained maximal $I_{\text {array }, B}(t)$ value.

Similarly we find the actual available charging current for the battery array, denoted by $I_{\text {array, }}^{a a}(t)$, at each time $t \in\left[T_{e}, T_{a}\right]$, based on the actual $G_{i}(t)$ and $T_{i}(t)(1 \leq i \leq$ $M)$ values. The actual available charging current $I_{\text {array,B }}^{a a}(t)$ is the actual maximum available battery array charging current, under the current solar irradiation and temperature. We also do not consider the supercapacitor array here. We 
obtain the $I_{a r r a y, B}^{a a}(t)$ value using Algorithm 1 (the B-VCTI algorithm) again, based on the current $G_{i}(t)$ and $T_{i}(t)$.

In the next step we compare the calculated $I_{\text {array,B }}^{\text {tar }}(t)$ and $I_{\text {array }, B}^{a a}(t)$ values. Generally speaking, the proposed HEES control algorithm make the supercapacitor array act as a buffer to store the excessive energy from PV modules when $I_{\text {array }, B}^{a a}(t)>I_{\text {array, } B}^{\text {tar }}(t)$, and provide energy to charge the battery array simultaneously with the PV modules when $I_{\text {array }, B}^{a a}(t)<I_{\text {array }, B}^{\text {tar }}(t)$. More precisely, there are three different cases based on the comparison results between $I_{\text {array, } B}^{\text {tar }}(t)$ and $I_{\text {array, } B}^{a a}(t)$, as follows, and we present the proposed near-optimal HEES control algorithm at time $t \in\left[T_{e}, T_{a}\right]$ in these three cases:

Case I $\left(I_{\text {array }, B}^{a a}(t)-I_{\text {array }, B}^{\text {tar }}(t)>\varepsilon\right)$ : This case is usually caused by a sudden peak of incoming PV power. In this case, we set the battery array charging current to be the target value $I_{\text {array,B }}^{\text {tar }}(t)$, and we maximize the supercapacitor array current $I_{\text {array, } C}(t)$ value by finding the optimal CTI voltage $V_{C T I}(t)$. To achieve this goal, we add a new control variable $I_{\text {array }, C}(t)$ to Algorithm 1 (the B-VCTI algorithm) for supercapacitor array power optimization, and set $I_{\text {array }, B}(t)=I_{\text {array }, B}^{\text {tar }}(t)$. We set $I_{\text {array, } C}(t)$ also to be the optimization objective of the modified B-VCTI algorithm.

Case II $\left(I_{\text {array,B }}^{a a}(t)-I_{\text {array }, B}^{\text {tar }}(t)<-\varepsilon\right)$ : This case is typically caused by a valley of incoming PV power. In this case, we set the battery array charging current to be the target value $I_{\text {array,B }}^{\text {tar }}(t)$, and we minimize the absolute value of $I_{\text {array, }}(t)$ by finding the optimal CTI voltage $V_{C T I}(t)$, since in this case we have $I_{\text {array, }}(t)<0$, i.e., the supercapacitor array is discharging. We adopt the modified B-VCTI algorithm used in Case I again in this optimization.

Case III $\left(\left|I_{\text {array }, B}^{a a}(t)-I_{\text {array }, B}^{\text {tar }}(t)\right|<\varepsilon\right)$ : In this case the difference between $I_{a r r a y, B}^{t a r}(t)$ and $I_{a r r a y, B}^{a a}(t)$ values is small. We set the battery array charging current to be the actual maximum available value $I_{\text {array,B }}^{a a}(t)$, and set the supercapacitor array current $I_{\text {array, } C}(t)$ to be zero.

The outline of the second step of the proposed HEES control algorithm is given in Algorithm 4. The MPTT and dynamic PV module reconfiguration methods can be effectively incorporated in this algorithm. We may replace the B-VCTI algorithm used in Algorithm 4 with the MVCTI, R-VCTI, or MR-VCTI algorithms without difficulty.

Algorithm 4: The outline of the second step of HEES control algorithm

At each time $t \in\left[T_{e}, T_{a}\right]$ :

Find $\widehat{G}_{i}(t)$ and $\widehat{T}_{i}(t)(1 \leq i \leq M)$ values using the crossover filter;

Find $I_{\text {array,B }}^{\text {tar }}(t)$ using the B-VCTI algorithm, based on $\widehat{G}_{i}(t)$ and $\widehat{T}_{i}(t)$;

Find $I_{\text {array, } B}^{a a}(t)$ using the B-VCTI algorithm, based on $G_{i}(t)$ and $T_{i}(t)$;

If $I_{\text {array, } B}^{a a}(t)-I_{\text {array }, B}^{\text {tar }}(t)>\varepsilon$ :

Set $I_{\text {array }, B}(t) \leftarrow I_{\text {array }, B}^{\text {tar }}(t)$; Find $I_{\text {array }, C}(t)$ using the modified B-VCTI algorithm; In this case $I_{\text {array, }}(t)>0$;

Else If $I_{\text {array,B }}^{a a}(t)-I_{\text {array, }}^{\text {tar }}(t)<-\varepsilon$ :

Set $I_{\text {array }, B}(t) \leftarrow I_{\text {array }, B}^{\text {tar }}(t)$; Find $I_{\text {array }, C}(t)$ using the modified

B-VCTI algorithm; In this case $I_{\text {array }, C}(t)<0$;

Else: Set $I_{\text {array }, B}(t) \leftarrow I_{\text {array }, B}^{a a}(t)$ and $I_{\text {array }, C}(t) \leftarrow 0$;

Until time $T_{a}$
3) Step Three:

The third and last step takes place during time period $\left[T_{a}, T_{d}\right]$. In this step, the charge stored in the supercapacitor array is migrated into the battery array. The motivation of this step is that our goal is to store all the solar energy in long-term storage devices, i.e., the battery array, by the deadline $T_{d}$, but there still exists residual energy in the supercapacitor array at time $T_{a}$, when the second step ends. Hence it is necessary to perform the charge migration during time period $\left[T_{a}, T_{d}\right]$ to maximize the energy conversion efficiency. According to [3], the charge migration problem in the third step is a time-constrained single-source singledestination charge migration problem with relative deadline $T_{d}-T_{a}$. This can be solved using the method proposed in [3]. Besides, we can derive the effective near-optimal solution by incorporating high-order curve fitting techniques with coefficients determined offline, with the benefit of reducing the online computational efforts.

\section{Experimental Result}

\begin{tabular}{|c|c|c|c|c|c|c|}
\hline $\begin{array}{c}\text { System } \\
\text { Index }\end{array}$ & $\begin{array}{c}\text { System } \\
\text { Architecture }\end{array}$ & $V_{C T I}(\mathrm{~V})$ & $\begin{array}{c}\text { Configuration } \\
m \times n\end{array}$ & $\begin{array}{l}\text { Tracking } \\
\text { Method }\end{array}$ & $\begin{array}{c}\text { Energy } \\
\text { into } \\
\text { Battery }(J) \\
\end{array}$ & $\begin{array}{c}\text { Normalized } \\
\text { Energy into } \\
\text { Battery }\end{array}$ \\
\hline 1 & \multirow{17}{*}{$\begin{array}{l}\text { Homo- } \\
\text { geneous }\end{array}$} & \multirow{3}{*}{5} & $5 \times 12$ & MPPT & $18,942.6$ & 0.8540 \\
\hline 1 & & & $10 \times 6$ & MPPT & $18,504.3$ & 0.8343 \\
\hline 1 & & & $15 \times 4$ & MIPPT & $16,739.1$ & 0.7547 \\
\hline 1 & & \multirow{3}{*}{10} & $5 \times 12$ & MPPT & $16,267.8$ & 0.7334 \\
\hline 1 & & & $10 \times 6$ & MIPPT & $17,678.7$ & 0.7970 \\
\hline 1 & & & $15 \times 4$ & MPPT & $17,300.5$ & 0.7800 \\
\hline 1 & & \multirow{3}{*}{15} & $5 \times 12$ & MPPT & $14,466.6$ & 0.6522 \\
\hline 1 & & & $10 \times 6$ & MPPT & $15,610.4$ & 0.7038 \\
\hline 1 & & & $15 \times 4$ & MPPT & $15,961.6$ & 0.7196 \\
\hline 2 & & \multirow{3}{*}{ Adaptive } & $5 \times 12$ & MPPT & $20,047.3$ & 0.9038 \\
\hline 2 & & & $10 \times 6$ & MPPT & $19,035.6$ & 0.8582 \\
\hline 2 & & & $15 \times 4$ & MPPT & $17,390.5$ & 0.7841 \\
\hline 3 & & \multirow{3}{*}{ Adaptive } & $5 \times 12$ & MPTT & $20,074.3$ & 0.9051 \\
\hline 3 & & & $10 \times 6$ & MPTT & $19,116.7$ & 0.8619 \\
\hline 3 & & & $15 \times 4$ & MPTT & $17,402.5$ & 0.7846 \\
\hline 4 & & Adaptive & Dynamic & MPPT & $20,428.6$ & 0.9210 \\
\hline 5 & & Adaptive & Dynamic & MPTT & $20,473.6$ & 0.9231 \\
\hline 6 & \multirow{8}{*}{ HEES } & \multirow{3}{*}{ Adaptive } & $5 \times 12$ & MPPT & $21,689.5$ & 0.9779 \\
\hline 6 & & & $10 \times 6$ & MPPT & $20,653.8$ & 0.9312 \\
\hline 6 & & & $15 \times 4$ & MPPT & $18,375.2$ & 0.8285 \\
\hline 7 & & \multirow{3}{*}{ Adaptive } & $5 \times 12$ & MPTT & $21,776.5$ & 0.9818 \\
\hline 7 & & & $10 \times 6$ & MPTT & $20,881.9$ & 0.9415 \\
\hline 7 & & & $15 \times 4$ & MPTT & $18,654.4$ & 0.8410 \\
\hline 8 & & Adaptive & Dynamic & MPPT & $22,052.6$ & 0.9943 \\
\hline 9 & & Adaptive & Dynamic & MPTT & $22,178.8$ & 1.0000 \\
\hline
\end{tabular}

Figure 8: Energy conversion efficiency simulation results

This section demonstrates the performances of the baseline system and the systems with different (combinations of) energy efficiency enhancing methods. We carry out experiments with timing parameters $T_{0}=0$, $T_{a}=3000 \mathrm{~s}$ and $T_{d}=3500 \mathrm{~s}$. The PV array consists of three PV modules, each consisting of 60 identical PV cells. The solar irradiation profile reflects partial shading in that each PV module may receive different solar irradiation. The temperature of the three PV modules is assumed to be a constant, which is the same as $T_{S T C}$ (temperature at standard test condition). The battery array in both the Homogeneous EES-Based System and the HEES-Based System has a nominal capacity of $1 \mathrm{Ah}$, and a nominal voltage of $8.4 \mathrm{~V}$ 
when the battery array is fully charged. The initial SoC of the battery array at time $T_{0}$ is set to $10 \%$. The capacitance of the supercapacitor array in the HEES-Based System is 100 F. We use Linear Technology LTM4607 converter as the charger with a modified feedback circuit, and obtain the parameters of the Li-ion battery model given in [12] by measurement. Experimental results are shown in Figure 8.

The System Index column in Figure 8 refers to the nine PV systems including the baseline system as annotated in Figure 5. The baseline system with system index of 1 cannot adjust the $V_{C T I}(t)$ value and the PV module configurations adaptively. The system with system index of 9 has all the proposed efficiency enhancement methods and yields a significant performance gain ranging from $17.1 \%$ to $53.3 \%$, compared with the baseline system.

We evaluate benefits and limitations of each proposed methods. The average benefit of an enhancement method implies average energy efficiency gain from only the specific method while keeping all other conditions the same. The average benefit is an effective metric that indicates the potential of efficiency improvement from the enhancement method. For example, the adaptive CTI voltage method exhibits average benefit of $12.6 \%$. This justifies that the system efficiency is highly dependent on the CTI voltage. The three advanced efficiency enhancement methods, i.e., HEES, MPTT, and dynamic PV module reconfiguration, exhibit average benefits of $7.95 \%, 0.60 \%$, and $9.07 \%$, respectively. The dynamic PV module reconfiguration has the highest potential in efficiency improvement. Although the MPTT shows minor performance enhancement in this experiment, it requires no additional hardware cost unlike the other two advanced methods. Moreover, the MPTT technique can be implemented just like the traditional MPPT. On the other hand, the dynamic PV module reconfiguration method requires additional hardware cost as shown in Figure 6 and also requires additional control algorithm for controlling the switches inside each PV module. The HEES enhancement method exhibits more consistent efficiency gain around 8\% compared to the dynamic PV module reconfiguration. Moreover, the HEES enhancement is also capable of providing efficiency improvement when a time-variant load is connected to the PV system, which is an additional advantage.

\section{Conclusion}

This paper addresses efficiency and robustness enhancement of standalone PV systems under partial shading. Partial shading is a major problem that causes severe efficiency degradation of PV systems in most locations. Use of an individual charger for each PV module may mitigate the negative effect from partial shading.

We see, however, such existing methods against partial shading do not provide an efficient design framework to accommodate electrical energy storage as well as charger efficiency variation. We introduce three methods to deal with partial shading of PV systems on the top of the individual charger architecture, which effectively overcome major drawbacks of existing systems. The three methods include i) incorporation of HEES (hybrid electrical energy storage) into the PV system and development of a near- optimal HEES control algorithm, ii) extension of the MPTT (maximum power transfer tracking), and iii) a dynamic PV module reconfiguration method. We show that the three methods can be effectively combined with each other and yield a significant energy conversion efficiency gain up to $53.3 \%$ compared with the baseline systems. We also present a design guideline based on the relative pros and cons of the three methods according to various operating conditions.

\section{References}

[1] M. Pedram, N. Chang, Y. Kim, and Y. Wang, "Hybrid electrical energy storage systems," in ISLPED, 2010.

[2] F. Koushanfar, "Hierarchical hybrid power supply networks," in DAC, 2010.

[3] Y. Wang, Y. Kim, Q. Xie, N. Chang, and M. Pedram, "Charge migration efficiency optimization in hybrid electrical energy storage (HEES) systems," in ISLPED, 2011.

[4] N. Femia, G. Petrone, G. Spagnuolo, and M. Vitelli, "Optimization of perturb and observe maximum power point tracking method," IEEE T. on Power Electronics, 2005.

[5] F. Liu, S. Duan, F. Liu, B. Liu, and Y. Kang, "A variable step size INC MPPT method for PV systems," IEEE T. on Industrial Electron., 2008.

[6] Y. Kim, N. Chang, Y. Wang, and M. Pedram, "Maximum power transfer tracking for a photovoltaicsupercapacitor energy system," in ISLPED, 2010.

[7] H. Patel and V. Agarwal, "Maximum power point tracking scheme for PV systems operating under partially shaded conditions," IEEE T. on Industrial Electronics, 2008.

[8] R. Bruendlinger, B. Bletterie, M. Milde, and H. Oldenkamp, "Maximum power point tracking performance under partially shaded PV array conditions," in Proc. of $21^{\text {st }}$ EUPVSEC, 2006.

[9] W. Xiao, N. Ozog, and W. G. Dunford, "Topology study of photovoltaic interface for maximum power point tracking," IEEE T. on Industrial Electron., 2007.

[10] W. Lee, Y. Kim, Y. Wang, N. Chang, M. Pedram, and S. Han, "Versatile high-fidelity photovoltaic module emulation system," in ISLPED, 2011.

[11] M. Chen and G. Rincon-Mora, "Accurate electrical battery model capable of predicting runtime and I-V performance," IEEE T. on Energy Conversion, 2006.

[12] D. Shin, Y. Wang, Y. Kim, J. Seo, M. Pedram, and N. Chang, "Battery-supercapacitor hybrid system for high-rate pulsed load applications," in DATE, 2011.

[13] D. Linden and T. D. Reddy, Handbook of Batteries, McGraw-Hill Professional, 2001. 